Fakultät III

Wirtschaftswissenschaften, Wirtschaftsinformatik und Wirtschaftsrecht

Volkswirtschaftliche Diskussionsbeiträge

Discussion Papers in Economics

No. $169-14$

November 2014

Gilbert Kollenbach

Endogenous growth with a limited fossil fuel extraction capacity 
Universität Siegen

Fakultät III

Wirtschaftswissenschaften, Wirtschaftsinformatik und Wirtschaftsrecht

Fachgebiet Volkswirtschaftslehre

Hölderlinstraße 3

D-57068 Siegen

Germany

http://www.wiwi.uni-siegen.de/vwl/

ISSN 1869-0211

Available for free from the University of Siegen website at

http://www.wiwi.uni-siegen.de/vwl/research/diskussionsbeitraege/

Discussion Papers in Economics of the University of Siegen are indexed in RePEc and can be downloaded free of charge from the following website:

http://ideas.repec.org/s/sie/siegen.html 


\title{
Endogenous growth with a limited fossil fuel extraction capacity
}

\author{
Gilbert Kollenbach ${ }^{\mathrm{a}, *}$ \\ ${ }^{a}$ Lehrstuhl für Volkswirtschaftslehre, insbesondere Finanzwissenschaft, Gilbert Kollenbach, 58084 Hagen, \\ Germany
}

\begin{abstract}
Campbell (1980) and following authors have discussed a limited resource extraction capacity as an augmentation of the well-known Hotelling model. We integrate a limited extraction capacity and related investments in the endogenous growth model of Tsur \& Zemel (2005) to study its effect on economic development. The capacity constraint gives raise to three effects. On the one hand, higher energy costs and the reallocation of production towards capacity investments decrease production available for consumption, research and/or general capital investments (energy cost and reallocation effect). On the other hand, research investments may increase, which boosts available production (research effect). Depending on the general capital endowment and the strength of the effects, long-run consumption may be boosted or depressed. In particular, the capacity constraint rationalizes the "curse of natural resources" phenomenon. Furthermore, we find that capacity investments may be postponed to later points in time, if the general capital endowment is very high.
\end{abstract}

Keywords: Exhaustible resources, limited extraction capacity, endogenous growth

JEL classification: Q32; Q42; O41

\section{Introduction}

The seminal work of Hotelling (Hotelling (1931)) has influenced many if not the most papers in the field of the economics of exhaustible resources. The famous Hotelling-rule, which states that the price of an exhaustible resource rises with the rate of interest, dates back to this work. However, empirical studies such as Gaudet (2007) and Livernois (2009) question the significance of this simple rule for observed price paths. ${ }^{1}$ A factor that explains deviations from the standard Hotelling-rule is a limited resource extraction capacity, which reflects the high capital intensity of mining. ${ }^{2}$ According to Cairns (1998), the discussion of limited resource extraction capacity dates back at least to Billiet (1959) and Massé (1959). The more recent literature strand begins with Campbell (1980). Among others, Cairns and Lasserre (1986), Cairns and Lasserre (1991), Davis and Moore (1998), Cairns (1998), Cairns (2001), Holland (2003), Ghoddusi (2010), and Lasserre (1985) belong to this strand. All mentioned studies focus on the optimal extraction of resources

\footnotetext{
*Department of Economics, University of Hagen, Universitätsstr. 41, 58097 Hagen, Germany, Telephone: +492331 987 - 2694, Fax: +492331 987 - 4143, University of Siegen, Germany

Email address: Gilbert.Kollenbach@Fernuni-Hagen.de (Gilbert Kollenbach)

${ }^{1}$ Cf. also Davis and Moore (1998) for further studies.

${ }^{2}$ Cf. Cairns (1998), page 234.
} 
and neglect other economic factors as general capital or research.

However, the endogenous growth theory considers research as a main driving force of economic growth. ${ }^{3}$ The impact of limited resources on endogenous growth is discussed by Barbier (1999), Schou (2000), and Tsur and Zemel (2005). ${ }^{4}$ To our knowledge no endogenous growth model considers a limited resource extraction capacity and a related investment possibility. The former affects resource extraction, while the latter competes with research and general capital for limited funds. To analyze the effects in detail, we augment Tsur and Zemel's (2005) suitable model with a limited resource extraction capacity and related investments. Exhaustible resources (fossil fuels) and a backstop resource (solar energy) enter the model as energy sources, so that the fossil fuel extraction period is finite. Due to the limited extraction capacity, fossil fuels are substituted by backstop ceteris paribus. On the one hand, this increases energy costs. On the other hand, more backstop utilization increases the advantageousness of research, as the backstop costs decrease in technology. Furthermore, capacity investments require a reallocation of production, so that less funds are available for consumption, research and/or general capital investments. We show under which conditions the three effects alter the optimal evolution path or the long-run position of the economy on the path. In particular, we rationalize the well-known "curse of natural resources" phenomenon. In contrast to the mentioned extraction capacity literature, we find that capacity investments may not be positive initially but postponed to later points in time, if the general capital endowment is very high.

The paper is structured as follows. The model is presented in section 2 and the conditions of the social optimum in section 3. Section 4 discusses the optimal economic evolution and the impact of the extraction capacity. Section 5 concludes.

\section{The Model}

The basis of our model, which is presented in the following, is the endogenous growth model of Tsur and Zemel (2005). ${ }^{5}$ The production of an universally usable final good $Y$ is based on general capital $K$ and energy $x$. The production function $F(K, x)$ is wellbehaved and concave, so that $F_{K}>0, F_{x}>0, F_{K K}<0, F_{x x}<0, F_{K x}=F_{x K}>0$, and $J:=F_{x x} F_{K K}-F_{x K}^{2}>0$. Capital is accumulated by saving final goods, whereas energy is generated by means of an exhaustible resource $R$ (fossil fuels) and an unlimited backstop $b$ (solar energy). The energy sources are perfect substitutes, so that $x=b+R$. The extraction costs of fossil fuels are given by the convexly increasing function $C(R)$, with $C_{R}>0$ and $C_{R R}>0$. The backstop utilization costs $B(A) b$ are linear in the backstop but declining in technology $A$, according to the convexly decreasing technology impact function $B(A)$. Thus, $B_{A}<0$ and $B_{A A}>0$. We assume that the cost decreasing effect of higher technology levels vanishes for large $A$, i.e. $\lim _{A \rightarrow \infty} B(A)=\bar{B}>0$. The positive technology endowment is denoted with $A(0) .{ }^{6}$ Technology increases in research

\footnotetext{
${ }^{3}$ The endogenous growth theory is reviewed by Aghion et al. (1998) and Barro and Sala-i Martin (2003).

${ }^{4}$ Pittel (2002) gives a comprehensive review of endogenous growth models with environmental concerns.

${ }^{5}$ For a detailed discussion of the assumptions we refer to Tsur and Zemel (2005). If not necessary for understanding, the time index $t$ is suppressed.

${ }^{6}$ The notation $X(0)$ is used for the initial values of all state variables.
} 
investments $a$ according to ${ }^{7}$

$$
\dot{A}=a \text {. }
$$

Fossil fuel extraction is constrained in two ways. The first constraint is given by the fossil fuel endowment $S(0)$, as $\int_{0}^{\infty} R(t) d t \leq S(0)$. The fossil fuel stock $(S)$ evolves in time according to

$$
\dot{S}=-R
$$

A limited fossil fuel extraction capacity imposes the second constraint on fossil fuel extraction. Following Campbell (1980), Davis and Moore (1998), Cairns (1998), and Cairns (2001), the extraction capacity is reflected by the specialized capital stock $Q .{ }^{8}$ One specialized capital unit allows the extraction of one fossil fuel unit at time $t$, so that

$$
Q(t)-R(t) \geq 0 \forall t
$$

As Cairns (1998) and Cairns (2001), we allow for capacity investments $q$ which increase extraction capacity according to

$$
\dot{Q}=q
$$

In contrast to $K$, the specialized capital is bound to the fossil fuel sector and immutable. Following Cairns and Lasserre (1986) and Tsur and Zemel (2005), we assume that there are no depreciations, and we impose an upper bound $\bar{I}$ on the investment sum $a+q . \bar{I}$ is given by net production

$$
Y^{n}:=F(K, x)-C(R)-B(A) b,
$$

i.e. by production net of energy costs. ${ }^{9}$ Net production is used for research, capacity investments, consumption $c$ and capital investments, so that

$$
\dot{K}=F(K, x)-C(R)-B(A) b-a-q-c .
$$

Consumption is the only argument of the concavely increasing utility function $U(c)$ of the representative individual. We apply the common assumptions $U_{c}>0, U_{c c}<0$ and $\lim _{c \rightarrow 0} U_{c}=\infty$.

\section{Social Optimum}

The social planner maximizes intertemporal utility $\int_{0}^{\infty} e^{-\rho t} U(c(t)) d t$, with $\rho$ denoting the time preference rate, subject to (1), (2), (4), (6), $\int_{0}^{\infty} R(t) d t \leq S(0)$, and the nonnegativity conditions (3), $R \geq 0, b \geq 0, a \geq 0, q \geq 0, c \geq 0$, and $\bar{I}-a-q \geq 0$. The current-value Lagrangian reads

$$
\begin{aligned}
L= & U(c)+\lambda[F(K, x)-C(R)-B(A) b-a-q-c]-\tau R+\kappa a+\theta q+\mu[Q-R] \\
& +\zeta_{R} R+\zeta_{b} b+\zeta_{a} a+\zeta_{q} q+\zeta_{c} c+\zeta_{\bar{I}}(\bar{I}-a-q) .
\end{aligned}
$$

\footnotetext{
${ }^{7}$ We define $\dot{z}:=\frac{d z}{d t}$ as the change of an arbitrary variable $z$ in time, and $\hat{z}:=\frac{1}{z} \frac{d z}{d t}$ as the growth rate.

${ }^{8}$ In the following we refer to $K$ as "capital" and omit "general". The latter is only used if needed to distinguish $K$ from $Q$.

${ }^{9}$ As shown below net production is not constant in time, so that $\bar{I}$ is variable.
} 
$\zeta_{i}, i=R, b, a, q, c, \bar{I}$ and $\mu$ denote the Lagrange multiplier of the non-negativity conditions. The shadow prices (costate variables) of the general capital stock, the fossil fuel stock, technology, and the specialized capital stock are $\lambda, \tau, \kappa$, and $\theta$. As an exogenous increase of the state variables is beneficial, or at least not harming, the shadow prices are nonnegative. Given an interior solution with respect to consumption, fossil fuel extraction and backstop use, the necessary conditions are ${ }^{10}$

$$
\begin{aligned}
U_{c}(c) & =\lambda, \\
F_{x}(K, x)=C_{R}(R)+\frac{\tau}{\lambda}-\frac{\mu}{\lambda} & =B(A), \\
-\lambda+\kappa+\zeta_{a}-\zeta_{\bar{I}} & =0 \\
-\lambda+\theta+\zeta_{q}-\zeta_{\bar{I}} & =0 .
\end{aligned}
$$

The growth rates of the shadow prices read

$$
\begin{aligned}
& \hat{\lambda}=\rho-F_{K}(K, x), \\
& \hat{\tau}=\rho, \\
& \hat{\kappa}=\rho-\frac{\lambda}{\kappa} b\left|B_{A}(A)\right|, \\
& \hat{\theta}=\rho-\frac{\mu}{\theta} .
\end{aligned}
$$

(8) and (12) give the well-known Ramsey rule

$$
\hat{c}=\frac{F_{K}-\rho}{\eta}
$$

with $\eta$ denoting the positive elasticity of marginal utility. If the marginal product of capital exceeds (falls short of) the time preference rate, consumption increases (declines). The absolute growth rate of consumption is the higher the smaller $\eta$.

The complement slackness condition with respect to fossil fuel capacity is given by

$$
\mu \geq 0, \mu[Q-R]=0
$$

The transversality conditions read ${ }^{11}$

$$
\begin{aligned}
& \text { (a) } \lim _{t \rightarrow \infty} e^{-\rho t} \lambda(t)\left[K(t)-K^{*}(t)\right] \geq 0, \quad(b) \lim _{t \rightarrow \infty} e^{-\rho t} \tau(t)\left[S(t)-S^{*}(t)\right] \geq 0, \\
& \text { (c) } \lim _{t \rightarrow \infty} e^{-\rho t} \theta(t)\left[Q(t)-Q^{*}(t)\right] \geq 0, \quad(d) \lim _{t \rightarrow \infty} e^{-\rho t} \kappa(t)\left[A(t)-A^{*}(t)\right] \geq 0 .
\end{aligned}
$$

Variables without an asterisk $(*)$ denote every feasible path and variables with an asterisk the optimal one.

(9) determines the energy mix, as illustrated in Fig. 1. Energy demand and supply

\footnotetext{
${ }^{10}$ We adopt Tsur \& Zemel's (2005) assumption of mixed energy generation. A positive consumption level in finite time follows from (8), (12), and the assumption $\lim _{c \rightarrow 0} U_{c}(c)=\infty$. According to (8), $\lambda$ equals $\infty$, if consumption decreases to zero. However, the growth rate of $\lambda$ is finite, as shown by (12), so that $\lambda(t)<\infty, \forall t<\infty$. The contradiction proves $c(t)>0, \forall t<\infty$.

${ }^{11}$ We use the transversality condition form of Feichtinger and Hartl (1986), page 42. Other dynamic optimization textbooks are Chiang (1992), Kamien and Schwartz (2000), and Seierstad and Sydsaeter (1987).
} 


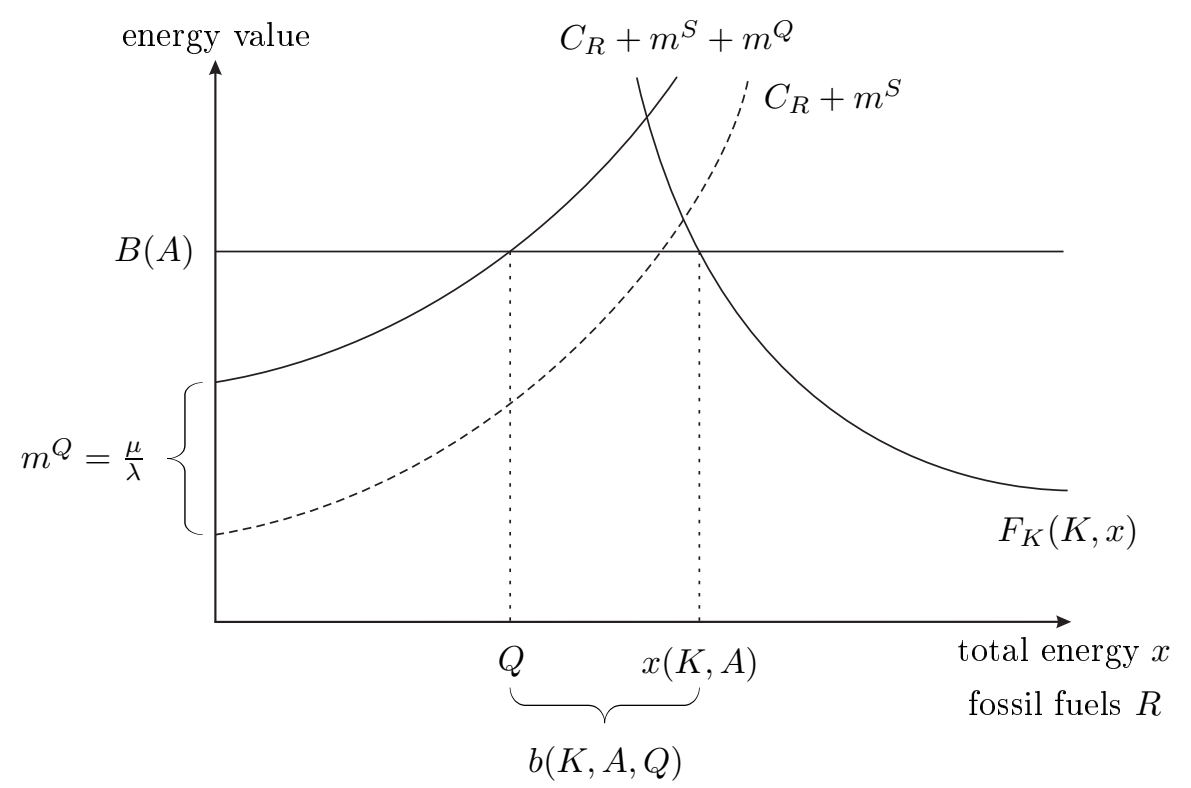

Figure 1: Determination of total energy supply $x$, backstop utilization $b$ and fossil fuel extraction $R$ by means of energy demand function $F_{K}(K, x)$, backstop supply function $B(A)$ and fossil fuel supply function $M_{R}(R)+m^{S}+m^{Q}$

of fossil fuels and backstop are given by the marginal product of energy $F_{x}(K, x)$, the marginal costs of backstop $B(A)$, and the marginal costs of fossil fuels $C_{R}(R)+\frac{\tau}{\lambda}+\frac{\mu}{\lambda}=$ $C_{R}(R)+m^{S}+m^{Q}$. The term $m:=m^{S}+m^{Q}=\frac{\tau+\mu}{\lambda}$ measures the scarcity of fossil fuels in relation to the scarcity of capital. Therefore, Kollenbach (2014a) refers to it as the relative scarcity index. It consists of the stock induced scarcity $m^{S}=\frac{\tau}{\lambda}$ and the capacity induced scarcity $m^{Q}=\frac{\mu}{\lambda}$. As we assume the mixed use of both energy sources, total energy input $x$ is determined by the intersection of the energy demand and the backstop supply function $F_{x}(K, x)=B(A)$, so that $x=x(K, A)$. If the extraction capacity constraint is not binding (dashed fossil fuels supply function), i.e. $m^{Q}=0$, the share of fossil fuels is given by $C_{R}(R)+m^{S}=B(A)$. Otherwise (solid fossil fuels supply function), $Q=R$ determines the share and $m^{Q}$ is such that $C_{R}(Q)+m^{S}+m^{Q}=B(A)$ holds. Thus, $R=R\left(A, m^{S}\right)$ or $R=R\left(A, m^{S}+m^{Q}\right)=Q$. Backstop supply equals the difference $b\left(K, A, m^{S}\right)=x(K, A)-R\left(A, m^{S}\right)$ or $b(K, A, Q)=x(K, A)-Q$. Differentiating $C_{R}(R)+m=B(A)$ and $F_{x}(K, x)=B(A)$ with respect to capital, technology and the relative scarcity index gives

$$
\frac{\partial R}{\partial A}=\frac{B_{A}}{C_{R R}}<0, \frac{\partial R}{\partial m}=-\frac{1}{C_{R R}}<0, \frac{\partial x}{\partial K}=-\frac{F_{x K}}{F_{x x}}>0, \frac{\partial x}{\partial A}=\frac{B_{A}}{F_{x x}}>0 .
$$

Due to $b(K, A, m)=x(K, A)-R(A, m)$, we get

$$
\frac{\partial b}{\partial A}>0, \frac{\partial b}{\partial K}>0, \frac{\partial b}{\partial m}>0 .
$$

Suppose the capacity constraint does not bind, so that $\mu=0$ and $\hat{m}=\hat{m}^{S}=F_{K}>0$. As $\frac{\partial R}{\partial m}<0, \frac{\partial R}{\partial A}<0$, and $\dot{A} \geq 0$, fossil fuel extraction decreases in time. Thus, if the capacity constraint becomes non-binding at time $t_{1}$, it will not bind for all $t \geq t_{1}$. Consequently, the constraint never binds, if it is non-binding at time $t=0$. Due to the continuous development of technology and stock induced scarcity, fossil fuel extraction 
is continuous at $t_{1} \cdot{ }^{12}$ Tsur and Zemel (2005) prove that fossil fuel extraction is also continuous at the time of fossil fuel exhaustion $(T)$.

If the capacity constraint binds, fossil fuel extraction is reduced below the level implied by the limited fossil fuel stock $\left(R\left(A, m^{S}\right)\right)$ and substituted by backstop. As the backstop costs are linear, whereas the extraction costs increase in current fossil fuel flow, the substitution increases energy costs. The cost increase is given by

$$
\omega=B(A)\left[R\left(A, m^{S}\right)-Q\right]-\left[C\left(R\left(A, m^{S}\right)\right)-C(Q)\right] .
$$

The first term on the right hand side measures the additional backstop costs and the second term the saved extraction costs of fossil fuels. Differentiating (21) with respect to technology and the stock induced scarcity gives

$$
\begin{aligned}
\frac{\partial \omega}{\partial A} & =B_{A}\left[R\left(A, m^{S}\right)-Q\right]+m^{S} \frac{\partial R\left(A, m^{S}\right)}{\partial A}<0, \\
\frac{\partial \omega}{\partial m^{S}} & =m^{S} \frac{\partial R\left(A, m^{S}\right)}{\partial m^{S}}<0 .
\end{aligned}
$$

Thus, the less developed the backstop technology and the lower the stock induced scarcity, i.e. the higher the fossil fuel stock, the larger is the ceteris paribus increase of energy costs. As apparent from Fig. 1, both is reflected by a larger capacity induced scarcity. According to (15), a large $\mu$ reduces the growth rate of the capacity shadow price. However, (18)(c), (15) and (17) require $\theta$ to be zero at the moment the capacity constraint becomes nonbinding. ${ }^{13}$ Thus, a higher $\mu$ allows for a higher initial capacity shadow price, which reflects a greater advantageousness of capacity investments.

To determine optimal investments we maximize $H=U(c)+\lambda[F(K, x)-C(R)-B(A) b$ $-a-q-c]-\tau R+\kappa a+\theta q$ with respect to $a$ and $q$. The result and the associated complement slackness conditions are presented in Appendix A and summarized in the following lemma.

\section{Lemma 1}

I Research investments are

(i) maximal, if $\kappa>\max [\lambda, \theta]$,

(ii) singular, if $\kappa=\lambda$ and $\kappa \geq \theta$,

(iii) minimal, if $\kappa<\max [\lambda, \theta]$.

II If net production is not completely used for research investments and $\theta>(=) \lambda$, capacity investments are maximal (singular). Otherwise, they are minimal.

III The sum of research and capacity investments is maximal, if $\kappa=\theta>\lambda$.

As $\theta(t)=0, \forall t \geq t_{1}$, parts II and III of lemma 1 are only relevant in the short-run. ${ }^{14}$ According to the lemma, short-run capacity investments are only positive, if the shadow

\footnotetext{
${ }^{12} \mathrm{~A}$ drop of extraction requires $\frac{\mu}{\lambda}<0$, which is ruled out by $\lambda>0$ and $\mu \geq 0$. An upward jump of extraction violates $Q \geq R$.

${ }^{13}$ Due to the exhaustibility of fossil fuels, the capacity constraint becomes non-binding at time $t_{1}$, so that $\mu=0$ and $\dot{\theta}=\theta \rho$. If $\theta>0$, its growth rate exceeds the ones of $\lambda$ and $\kappa$. Consequently, fossil fuel capacity investments are positive for large $t$. Thus, $\lim _{t \rightarrow \infty}\left[Q(t)-Q^{*}(t)\right]$ can be negative. Since $\theta>0$, we get a contradiction to $(18)(\mathrm{c})$.

${ }^{14}$ The long-run evolution and the mechanism determining both short-run and long-run development are discussed in section 4.
} 
price of capacity exceeds or, at least, equals the shadow prices of capital and research. Simultaneous capacity and research (capital) investments over a non-marginal time period require $\theta=\kappa(\theta=\lambda)$. From (15) and (14) ((12)) we get $\mu=\lambda b\left|B_{A}(A)\right|\left(\mu=\lambda F_{K}(K, x)\right)$. If the shadow price of capacity initially exceeds the other two shadow prices, capacity investments are maximal. As $\theta\left(t_{1}\right)=0$, capital and research investments become relatively more favorable in time. Thus, capacity investments vanish at least directly before $t_{1}$, a result also obtained by Cairns and Lasserre (1986) and Cairns (1998). ${ }^{15}$ The advantageousness of capacity investments is decreased by the increasing stock induced scarcity and possible research investments. Ceteris paribus, both reduce the capacity induced reduction of fossil fuel extraction. Under specific conditions capacity investments are zero initially but positive later on.

Proposition 1 If the capital endowment of the economy is very high, the capacity induced reduction of fossil fuel small, the technology impact function inelastic, the capacity constraint binding, and $\kappa>\lambda$, capacity investments may be minimal initially but positive during a later limited time period.

The first two requirements imply both a small $F_{K}$ and a small $\mu$, so that the growth rate of the stock induced scarcity $m^{S}$ is low, while the growth rates of $\lambda$ and $\theta$ approximate $\rho$. Thus, capacity investments may become more favorable than research investments, as $\hat{\theta}>\hat{\kappa} .{ }^{16}$ A declining growth rate of $\theta$ at later points in time is ensured by the inelastic technology impact function and the high capital endowment. On the one hand, the growth rate of the stock induced scarcity $\hat{m}^{S}=F_{K}$ is small. On the other hand, research decreases backstop costs only to a small extend. Thus, the capacity induced scarcity $m^{Q}=\frac{\mu}{\lambda}$ slightly decreases in time. As $\lambda$ grows nearly with the rate $\rho$, the growth rate of $\mu$ need to approximate $\rho$, too. Given that $\mu$ grows faster than $\theta, \frac{d \hat{\theta}}{d t}<0$.

Proposition 1 contrasts with Cairns (1998) and the other capacity literature. The disparity is explained by backstop and research. Due to the high capital endowment, backstop utilization is high at early points in time, so that maximal research is favorable. However, maximal research decreases the capital stock, which lowers backstop utilization. If the net effect of $\dot{A}>0$ and $\dot{K}<0$ on backstop use is negative, whereas $R=Q$, the relative advantageousness of capacity investments increases.

The two possible fossil fuel extraction paths are depicted in Fig. 2. The path on the left hand side illustrates conventional fossil fuel extraction in presence of a capacity constraint and limited investments possibilities. The capacity constraint binds during the period $\left[0, t_{1}\right]$, so that capacity investments are positive at early points in time. As $R(t)=Q(t)$, fossil fuel extraction increases. As soon as either capital or research investments become more favorable, fossil fuel extraction reaches its peak to continuously decrease to zero after the constraint becomes non-binding. If proposition 1 holds, an initial phase of constant fossil fuel extraction is added to the extraction path, as illustrated on the right hand side of Fig. 2.

\footnotetext{
${ }^{15}$ Let $^{-}\left({ }^{+}\right)$denote values directly before (after) $t_{1}$. As the state variables $K$ and $A$ are continuous, $F_{x}\left(K^{-}, x\left(K^{-}, A^{-}\right)\right)=F_{x}\left(K^{+}, x\left(K^{+}, A^{+}\right)\right)$. From (9) we get $C_{R}\left(R^{-}\right)+\frac{\tau^{-}}{\lambda^{-}}+\frac{\mu^{-}}{\lambda^{-}}=C_{R}\left(R^{+}\right)+\frac{\tau^{+}}{\lambda^{+}}$. According to (12) and (13), $\frac{\tau^{-}}{\lambda^{-}}=\frac{\tau^{+}}{\lambda^{+}}$, so that $C_{R}\left(R^{-}\right)+\frac{\mu^{-}}{\lambda^{-}}=C_{R}\left(R^{+}\right)$. Since $R^{-}=Q, \mu^{-}>0$ requires $C_{R}(Q)<C_{R}\left(R^{+}\right)$, i.e. $R^{+}>Q$. As this contradicts $Q \geq R, \mu^{-}=0$ and therefore $\theta^{-}=0$.

${ }^{16} \mathrm{It}$ is shown below that research is superior to capital investments, if the capital stock is high.
} 

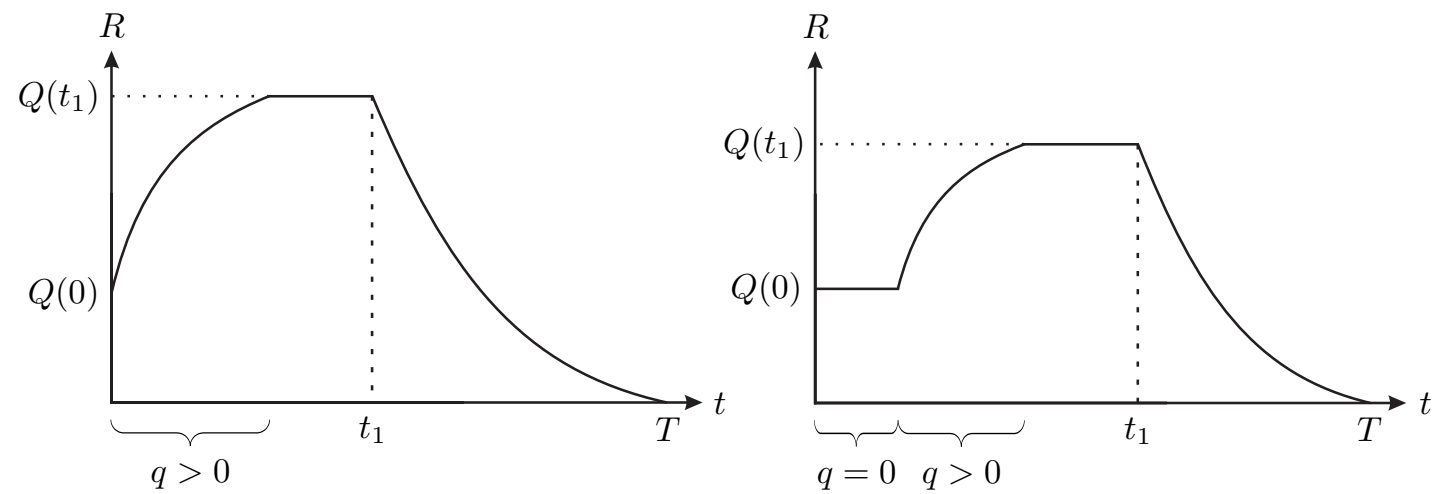

Figure 2: Fossil fuel extraction paths with and without initial capacity investments

\section{Economic evolution}

To determine the evolution of the economy, we apply the method developed by Tsur and Zemel (2005). ${ }^{17}$ They show that evolution depends on the position of the economy, characterized by the current capital stock and technology level, in the technology-capital space in relation to two characteristic lines. The evolution process can be illustrated as a path in the $(A, K)$ space, which is subsequently called the $(A, K)$ process.

\subsection{The characteristic lines}

The singular line characterizes all points of the $(A, K)$ space that may allow for singular research, while the steady-state line is defined by the steady-state $\hat{C}=\hat{K}=\hat{A}=$ $\hat{Q}=0 .{ }^{18}$ Following Tsur and Zemel (2005), we assume that both lines are non-decreasing. The lines divide the $(A, K)$ space into subspaces with the following specific properties. ${ }^{19}$

a) If not located on one of the lines, an $(A, K)$ process approaches either the singular or the steady-state line with minimal or maximal research investments. Thus, the $(A, K)$ process follows a most rapid research approach path (MRRAP).

b) Above the singular line research investments are either minimal or maximal, while they are minimal below the line. According to lemma $1, \kappa<\lambda$ below the line but either $\kappa>\lambda$ or $\kappa<\lambda$ above it.

c) The singular line bounds an $(A, K)$ process. In other words, an $(A, K)$ process has to stay in the singular line forever after it has reached the line. Thus, if the $(A, K)$ process reaches the singular line at time $t_{z}, \kappa(t)=\lambda(t), \forall t \geq t_{z}$.

\footnotetext{
${ }^{17}$ We refer to Tsur and Zemel (2005) for details and proofs. Cf. also Kollenbach (2014a).

18 The singular line, which is described by the function $K^{N}(A, m)$, is implicitly given by $F_{K}(K, x(K, A))=-B_{A}(A) b(K, A, m)$. Thus, there is a line for every $m$. As we can assign every $m$ a time $t$, the set of lines forms a manifold in the $(A, K, t)$ space. According to $(16), F_{K}(K, x(K, A))=\rho$ needs to hold in a steady-state. The equation implicitly defines the steay-state line, which is given by the function $K^{S}(A)$. Tsur and Zemel (2005) show that both lines are non-decreasing for large $A$.

${ }^{19} \mathrm{We}$ use the formulation "the $(A, K)$ process is below/above/on the singular/steady-state line" to indicate that for the current technology value the capital value of the $(A, K)$ process is smaller than/larger than/equal to the capital value of the respective line. Furthermore, if the $(A, K)$ process reaches the singular line at time $t$, it always reaches the projection of time $t$ of the singular line manifold, mentioned in footnote 18 , on the $(A, K)$ space.
} 
d) Consumption increases (decreases), if the $(A, K)$ process is located below (above) the steady-state line. Due to (16), $F_{K}>\rho$ below and $F_{K}<\rho$ above the line.

According to properties a) and $\mathrm{c}$ ), the $(A, K)$ process is located either on the singular line or the steady-state line in the long-run. As consumption decreases above the steadystate line, long-run evolution along the singular line is only possible, if the singular line is located below the steady-state for large $A$. Therefore, Tsur and Zemel (2005) refer to this economy type as a (potentially) growing economy. ${ }^{20}$ If the singular line is located above the steady-state line for large $A$, the $(A, K)$ process converges towards the steadystate line in the long-run. Otherwise, consumption would decrease continuously. The corresponding economy type is referred to as a converging economy.

\subsection{Scarcity and optimal evolution}

According to (20), backstop utilization increases with the relative scarcity index. Ceteris paribus, research investments become more advantageousness. This requires a lower position of the singular line in the $(A, K)$ space, as research investments can be positive only on or above the line. Thus, the singular line shifts downwards in the $(A, K)$ space, if the relative scarcity index increases. ${ }^{21}$ Suppose the capacity constraint never binds. The relative scarcity index grows continuously with the rate $\hat{m}=F_{K}$, so that the singular line shifts downwards until the fossil fuel stock is exhausted at time $T$. For all $t \geq T$, the position of the singular line does not change, as the economy relies only on backstop. Note that the capacity constraint does not alter the long-run position of the singular line. ${ }^{22}$ Thus, the constraint does not affect the type (potentially growing, converging) of the economy.

If the capacity constraint binds for the limited time period $\left[t_{0}, t_{1}\left[\right.\right.$, with $0 \leq t_{0}<t_{1}$, the binding constraint links the evolution of the relative scarcity index to capacity and research investments. As evident from Fig. 1, the relative scarcity index, and therefore the advantageousness of research, remains constant in time, if capacity and research investments are minimal. In case of positive research investments, backstop costs are lowered. To ensure $R=Q$, the relative scarcity index needs to decrease. ${ }^{23}$ If capacity investments are positive, $d Q>0$ and $R=Q$ require also a decrease of the relative scarcity index. ${ }^{24}$ Both research and capacity investments reduce the capacity induced substitution of fossil fuels by backstop. Consequently, both reduce the advantageousness of research. To sum up, research advantageousness

- decreases, if the capacity constraint is binding and research and/or capacity investments are positive,

- is constant, if the capacity constraint is binding and research and/or capacity investments are minimal, or if the fossil fuel stock is exhausted,

- increases, if the capacity constraint is non-binding but the fossil fuel stock not exhausted.

\footnotetext{
20 "Potentially" will be explained below.

${ }^{21}$ From footnote 18 we get $\frac{d K^{N}}{d m}<0$ and $\frac{d K^{S}}{d m}=0$.

${ }^{22}$ This follows from the definition of the singular line. If the economy only relies on backstop, the $(A, K)$ combinations solving $F_{K}(K, x(K, A))=-B_{A}(A) b$ (from footnote 18) are independent form the capacity constraint.

${ }^{23}$ This follows from $\dot{A}>0, B_{A}(A)<0, \frac{\partial R}{\partial A}<0$, and $\frac{\partial R}{\partial m}<0$, too.

$24 \frac{\partial R}{\partial m}<0$ gives the same result.
} 
Graphically, this is illustrated in Fig. 3 by the arrows between the singular line of the initial time $(S i L(0))$ and the singular line of fossil fuel exhaustion time $(S i L(T))$. The left arrow, marked with $t_{1}$, refers to the time period of a binding capacity constraint $\left[t_{0}, t_{1}[\right.$ and the right arrow to all other points in time. A dash on top of an arrow indicates that the singular line may not change its position during time.

At every point in time a singular line $S i L(t)$ exists. The set of lines forms a manifold in the $(A, K, t)$ space, with $S i L(t)$ as the projection of the manifold on the $(A, K)$ space at time $t$. In the following analysis of the optimal economic evolution we will say that "the $(A, K)$ process reaches the singular line at time $t$ ". We always refer to the current projection of the manifold, i.e. to $S i L(t) .{ }^{25}$ In contrast to the singular line, the position of the steady-state line in the $(A, K)$ space is not affected by scarcity. ${ }^{26}$

On the left hand side of Fig. 3 we illustrate three exemplary $(A, K)$ processes of a potentially growing economy and on the right hand side three exemplary processes of a converging economy. The processes are identified by their capital endowments $K^{i}, i=1, \ldots, 4{ }^{27}$ The capacity induced substitution of fossil fuels by backstop and the correspondingly larger research advantageousness may affect each process in two opposing ways. On the one hand, a higher utilization of backstop ceteris paribus increases energy costs by $\omega$ and therefore decreases net production. Thus, a negative energy cost effect decreases the level of consumption or investments. The effect is the stronger the larger $\omega$.

On the other hand, the higher research advantageousness may induce more research investments. According to $B_{A}(A)<0$ and (19), a higher technology level decreases energy costs and boosts total energy use. Both increase net production and therefore consumption and/or investments. We refer to this mechanism as the research effect. ${ }^{28}$

As capacity investments reduce the tightness of the capacity constraint, they mitigate both effects. However, capacity investments require a reallocation of net production. Consequently, a reallocation effect lowers available production for consumption, capital and/or research investments. ${ }^{29}$

In case of the $K_{1}$ path, we assume that net production is initially used for maximal capacity investments. Consequently, the capital stock decreases while the technology level remains constant. As the advantageousness of capacity investments vanishes in the long-run, investments switch from capacity to research and/or capital after some time. ${ }^{30}$ As the capital endowment is large, we assume that the $(A, K)$ process is located above the singular line at the switching time, so that the $(A, K)$ process approaches the singular line with maximal research investments. After the singular line is reached, the

\footnotetext{
${ }^{25}$ See also footnote 18 .

${ }^{26}$ This follows directly from the defining function $F_{K}(K, x(K, A))=\rho$, which depends on capital and technology but is independent from the relative scarcity index.

${ }^{27}$ For each path we make assumptions about the point in time the $(A, K)$ process reaches the singular line, the length of the capacity investments period, and the first point in time capacity investments are positive. These assumptions are only made for illustration purposes. Different assumptions imply alternative evolution paths.

${ }^{28}$ According to Kollenbach (2014a), a ceiling on the stock of pollution gives also rise to an energy cost and a research effect.

${ }^{29}$ As the capacity constraint restricts the social planner, the energy cost and reallocation effect dominate the research effect with respect to welfare.

${ }^{30}$ According to lemma 1, there can be a period of shared investments. However, the illustration would not be changed qualitatively. Therefore, this case is neglected here.
} 

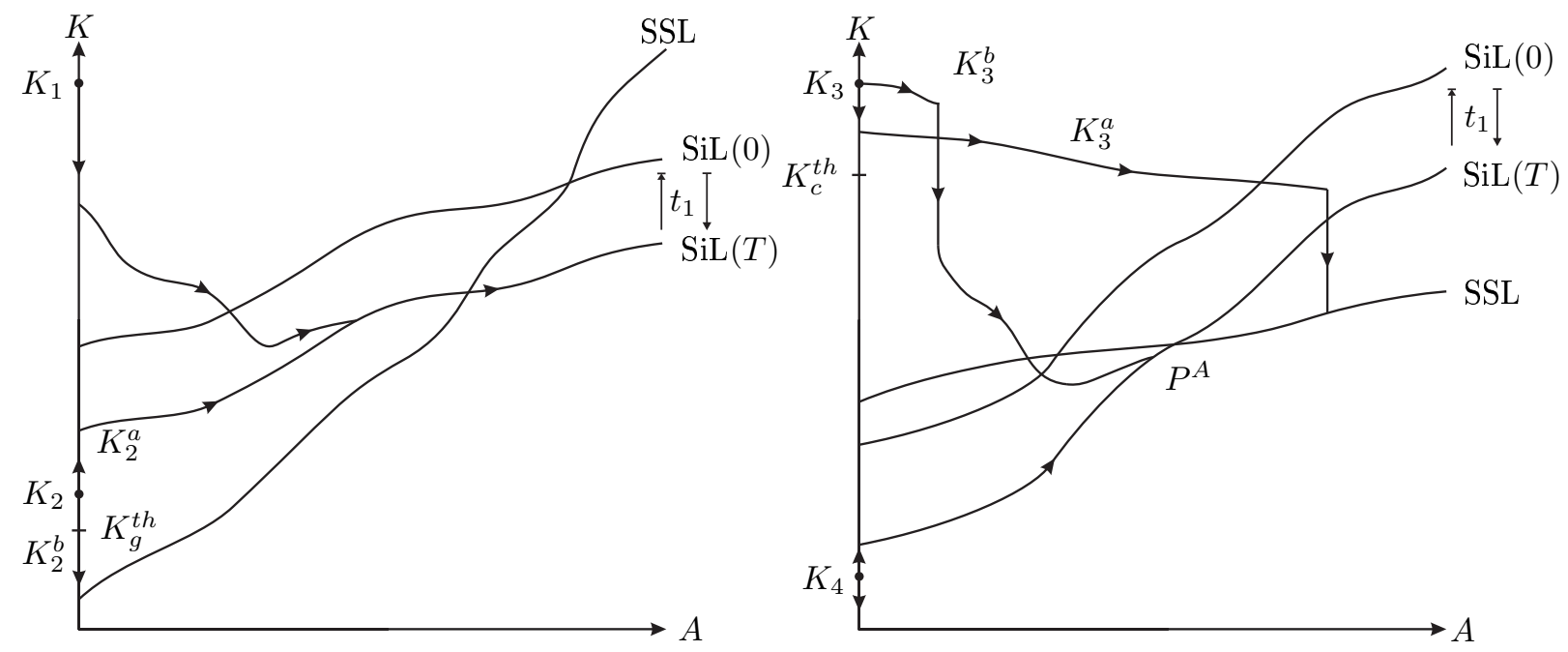

Figure 3: Six exemplary evolution processes of a (potentially) growing and a converging economy in the $(A, K)$ space determined by the steady-state line (SSL) and the singular line (SiL), the latter illustrated for $t=0$ and $t=T$. Arrows indicate the direction of the evolution process.

economy grows along it forever. ${ }^{31}$ As an economy with the capital endowment $K_{1}$ but without an extraction capacity constraint would have grown along the long-run singular line $(\operatorname{SiL}(T))$, the energy costs, the reallocation, and the research effect do not alter the long-run evolution path. However, the position of the $(A, K)$ process on the evolution path at some point in time is altered. As the first two effects decrease available production, they slow down the evolution along the path. In contrast, the research effect increases available production. If the energy cost and reallocation effects dominate, capital stock and technology levels are lower at some point in time $t>T$ than without the capacity constraint. According to

$$
d F_{K}=J d K+\frac{F_{K x}}{F_{x x}} B_{A}(A) d A<0
$$

and (16), the consumption growth rate and consequently long-run consumption is decreased.

We illustrate two alternative evolution paths with the capital endowment $K_{2}$. The threshold $K_{g}^{t h}$ is crucial to understand the two paths. If the capital endowment of an economy without an extraction capacity constraint does not exceed the threshold, it is too poor to invest in research or capital. ${ }^{32}$ In other words, only an economy that has a sufficiently large capital endowment can afford a temporary lower consumption level necessary for investments. Both the $K_{2}^{a}$ and the $K_{2}^{b}$ path start with the capital endowment $K_{2}>K_{g}^{t h}$. In case of the $K_{2}^{a}$ path, capacity investments may be maximal at early points in time but capital investments become at least as favorable as capacity investments before the $(A, K)$ process has reached the threshold $K_{g}^{t h}$. Thus, capital is accumulated and the $(A, K)$ pro-

\footnotetext{
${ }^{31}$ The uptick in the evolution path indicates that the $(A, K)$ process reaches the singular line before the exhaustion of fossil fuel.

32 The threshold $K_{g}^{t h}$ need not to be identical with the threshold of an economy with a capacity constraint. However, distinguishing between two threshold complicates the illustration without providing much more insight. Therefore, we assume the identity of the two thresholds.
} 
cess approaches the singular line to evolve along it forever. As in case of the $K_{1}$ path, the strength of the reallocation, energy costs and research effect determines if long-run consumption is increased or decreased.

In the contrary case of the $K_{2}^{b}$ path, the threshold $K_{g}^{\text {th }}$ is reached due to a strong reallocation effect, i.e. due to a high advantageousness of capacity investments. Consequently, capital and later research investments are unaffordable and the $(A, K)$ process converges against a steady-state with $A(t)=A(0), \forall t$. Thus, the capacity constraint may lead the economy into a poverty trap. Nonetheless, the $K_{2}^{b}$ path illustrates a possible optimal evolution path. To be optimal the short-run consumption development on the $K_{2}^{b}$ path needs to compensate for the unused growth option, i.e. for low long-run consumption. This requires a strong effect of capacity investments on net production at early points in time, i.e. a large $\omega$. As explained above, $\omega$ is the larger the higher the fossil fuel endowment $S(0)$. Thus, ignoring the growth option due to a capacity constraint and a high fossil fuel endowment rationalizes the "curse of natural resources" phenomenon. Tsur and Zemel (2005) also refer to the curse. However, they compare a resource abundant economy with an economy with a limited resource stock. In contrast, we refer only to economies with limited but differently large resource stocks. Thus, we show that not only an unlimited fossil fuel stock may give rise to the "curse of natural resources" but also an exhaustible stock in combination with limited extraction capacities.

For the two evolution paths starting with the capital endowment $K_{3}$ we have to take the threshold $K_{c}^{\text {th }}$ into account. ${ }^{33}$ If the capital endowment of a converging economy without a capacity constraint exceeds $K_{c}^{t h}$, high research investments prevent a convergence against the steady-state at the intersection $P^{A}$ of the steady-state line and the long-run singular line $(\operatorname{SiL}(T))$. Thus, the evolution path can only reach a part of the singular line that is located above the steady-state line. According to property d), consumption decreases in this area. As consumption is constant in a steady-state, a position on the steady-state line is superior to an evolution along the singular line. Thus, an optimal $(A, K)$ process abandons maximal research before the singular line is reached, so that the process converges against the steady-state line. Qualitatively, this case is illustrated by the $K_{3}^{a}$ path. We assume that a switch from initially positive capacity investments to research occurs at an early point in time, so that the $(A, K)$ process leads to a steady-state to the right of $P^{A}$. If maximal research is abandoned before $t_{1}$, the singular line is located below the position implied by the stock induced scarcity. This may allow more research than in an economy without the capacity constraint, i.e. a strong research effect. Consequently, the reached steady-state exhibits a higher level of capital and of technology, which both boosts production. Due to (6) and $\dot{K}=0$ in a steady-state, steady-state consumption is increased.

With the $K_{3}^{b}$ path we illustrate the above-mentioned case of an economy with mid-term capacity investments. ${ }^{34}$ While the capital endowment is high and research investments maximal initially, capacity investments become more favorable for a limited time period. If the reallocation effect is sufficiently strong, the capital stock decreases for a longer time period, so that the $(A, K)$ process reaches the singular line to the left of $P^{A}$ to converge against this steady-state. Otherwise, a steady-state to the right of $P^{A}$, which exhibits

\footnotetext{
${ }^{33}$ As in case of the threshold $K_{g}^{t h}$, we assume that $K_{c}^{t h}$ is also valid for the illustrated evolution path. Cf. footnote 32 .

${ }^{34}$ Recall that a requirement for mid-term capacity investments was an inelastic research impact function. If this requirement is not met, the economy may develop as illustrated by the $K_{3}^{a}$ path.
} 
a higher consumption level, would be reached. Consequently, the benefits of capacity investments, in terms of lower energy costs which increase consumption, need to offset for lower long-run consumption. This short-term consumption increase can be realized after the $(A, K)$ process has reached the singular line. As fossil fuel is still used at this time, singular research investments and a capacity induced reduction of fossil fuel costs allow an increase of consumption.

The capital endowment of the $K_{4}$ path is low, so that the $(A, K)$ process approaches the singular line from below after an initially phase of maximal capacity investments. Since the $(A, K)$ process converges against the steady-state $P^{A}$ with and without the capacity constraint, the latter does not alter the long-run evolution of the economy. As in case of the $K_{1}$ path, sufficiently strong energy costs and reallocation effects slow down the evolution along the path, so that consumption growth during the convergence process is decreased. The above discussion of the evolution paths is summarized in proposition 2 .

Proposition 2 (i) Suppose the fossil fuel endowment of a potentially growing economy is sufficiently large. Then, the benefits of capacity investments outweigh the ones of everlasting growth, so that the growth option is ignored. Otherwise, a dominant research effect increases long-run consumption.

(ii) If the capital endowment of a converging economy is sufficiently large, the capacity constraint may increase or decrease steady-state consumption. Otherwise, steady-state consumption is not affected, but a dominant research effect increases consumption during the convergence process.

According to Kollenbach (2014a) and Kollenbach (2014b), a pollution problem, represented by polluting fossil fuels and a ceiling on the stock of pollution $\left(\mathrm{CO}_{2}\right)$, give raise to similar results. However, we abstract from environmental concerns, while a limited fossil fuel extraction capacity and the related specialized capital stock are not considered by Kollenbach (2014a) and Kollenbach (2014b). Similar to our $K_{2}^{b}$ path, Kollenbach (2014b) finds that abatement activities may lead an economy into the poverty trap. The common feature of both cases is a reallocation of net production which relaxes a constraint on fossil fuel extraction. In case of Kollenbach (2014b), abatement activities allow more fossil fuel extraction without violating a pollution ceiling, while in our case capacity investments increase a bounded extraction rate.

\section{Conclusion}

The literature strand following Campbell (1980) augments the well-known Hotelling model with a limited resource extraction capacity to reflect the high (specialized) capital intensity of the mining industry and to improve the consistency of theory and empirical findings. We incorporate the limited capacity assumption in Tsur \& Zemel's (2005) endogenous growth model to study the effects on economic development. If the economy has the potential for everlasting growth and a high fossil fuel endowment, the capacity constraint may rationalize the "curse of natural resources" phenomenon. In this case, capacity investments, which lower the energy costs, are more favorable than (general) capital and research investments, so that the growth option is ignored. If the growth option is realized, the capacity constraint affects the economic development in three ways. On the one hand, higher energy costs (energy cost effect) and the reallocation of net production in favor of capacity investments (reallocation effect) decrease available production and therefore slow down development. On the other hand, a higher ceteris paribus utilization of 
backstop may increase research investments, which increase available production (research effect). Given that the research effect dominates, long-run consumption is increased. In case of a converging economy with a high capital endowment, a sufficiently strong reallocation effect decreases steady-state consumption. However, steady-state consumption is increased, if the reallocation effect is weak and the research effect sufficiently strong. The latter increases also the consumption level during the convergence process against the unchanged steady-state of a converging economy with a low capital endowment.

Our results complement the ones of Tsur and Zemel (2005), Kollenbach (2014a) and Kollenbach (2014b). Thus, Tsur and Zemel (2005) refer also to the "curse of natural resources". However, their benchmark case is a resource abundant economy, while our one is an economy with a limited resource stock but without a capacity constraint. Kollenbach (2014a) and Kollenbach (2014b) find that environmental concerns, such as a ceiling on $\mathrm{CO}_{2}$ emissions, give also raise to an energy costs and a research effect. However, a capacity constraint is not considered.

In contrast to the capacity literature, e.g. Campbell (1980) and Cairns (1998), we find that capital, research and backstop may delay capacity investments. In this case, a very large capital endowment implies a high backstop utilization and therefore the initially superiority of maximal research investments. Given that the capital stock decreases sufficiently fast, the energy mix share of fossil fuels increases, so that capacity investments become favorable at a later point in time.

To focus on the effect of the capacity constraint, we have abstained from environmental concerns, different fossil fuels, and technological progress in other sectors than the backstop one. Incorporating fossil fuel caused pollution $\left(\mathrm{CO}_{2}\right)$ would cause cross-effects between the capacity constraint and the environmental concerns, as both reduce fossil fuel extraction ceteris paribus. By introducing research in the fossil fuel sector, it would be possible to analyze the effect of a capacity constraint on the direction of technical change. ${ }^{35}$

\section{References}

Acemoglu, D., 2002. Directed technical change. Review of Economic Studies 69 (4), 781809.

Acemoglu, D., Aghion, P., Bursztyn, L., Hemous, D., 2012. The Environment and directed technical change. American Economic Review 102 (1), 131-166.

Aghion, P., Howitt, P., García-Peñalosa, C., 1998. Endogenous growth theory. the MIT Press.

Barbier, E., 1999. Endogenous growth and natural resource scarcity. Environmental and Resource Economics 14 (1), 51-74.

Barro, R., Sala-i Martin, X., 2003. Economic growth, 2nd Edition. The MIT Press.

Billiet, A., 1959. Sur la Recherche de l'Optimum d'Exploitation d'un Gisement Minier de Capacité Incertaine. I. Avenir certain: Revue Française de Recherche Opérationnelle (10), 21-24.

\footnotetext{
${ }^{35}$ Cf. Acemoglu (2002) and Acemoglu et al. (2012).
} 
Cairns, R. D., 1998. The microeconomics of mineral extraction under capacity constraints. Nonrenewable resources 7 (3), 233-244.

Cairns, R. D., 2001. Capacity Choice and the Theory of the Mine. Environmental and Resource Economics 18 (1), 129-148.

Cairns, R. D., Lasserre, P., 1986. Sectoral supply of minerals of varying quality. The Scandinavian Journal of Economics, 605-626.

Cairns, R. D., Lasserre, P., 1991. The role of investment in multiple-deposit extraction: Some results and remaining puzzles. Journal of Environmental Economics and Management 21 (1), 52-66.

Campbell, H. F., 1980. The effect of capital intensity on the optimal rate of extraction of a mineral deposit. Canadian Journal of Economics, 349-356.

Chiang, A., 1992. Elements of dynamic optimization. McGraw-Hill Companies.

Davis, G. A., Moore, D. J., 1998. Valuing mineral reserves when capacity constrains production. Economics Letters 60 (1), 121-125.

Feichtinger, G., Hartl, R., 1986. Optimale Kontrolle ökonomischer Prozesse: Anwendungen des Maximumprinzips in den Wirtschaftswissenschaften. Walter de Gruyter.

Gaudet, G., 2007. Natural resource economics under the rule of Hotelling. Canadian Journal of Economics/Revue canadienne d'économique 40 (4), 1033-1059.

Ghoddusi, H., 2010. Dynamic investment in extraction capacity of exhaustible resources. Scottish Journal of Political Economy 57 (3), 359-373.

Holland, S. P., 2003. Extraction capacity and the optimal order of extraction. Journal of Environmental Economics and Management 45 (3), 569-588.

Hotelling, H., 1931. The economics of exhaustible resources. Journal of Political Economy 39, 137-175.

Kamien, M., Schwartz, N., 2000. Dynamic optimization: the calculus of variations and optimal control in economics and management, 2nd Edition. North-Holland New York.

Kollenbach, G., 2014a. Endogenous Growth with a Ceiling on the Stock of Pollution. Einvironmental and Resource Economics DOI: 10.1007/s10640-014-9832-6.

Kollenbach, G., 2014b. Research or Carbon Capture and Storage - How to limit climate change? Tech. rep., University of Siegen, Discussion Papers in Economics No. 163-14.

Lasserre, P., 1985. Capacity choice by mines. Canadian Journal of Economics, 831-842.

Livernois, J., 2009. On the empirical significance of the Hotelling rule. Review of Environmental Economics and policy 3 (1), 22-41.

Massé, P., 1959. Le choix des investissements. Dunod Paris, pp. 348-353.

Pittel, K., 2002. Sustainability and endogenous growth. Edward Elgar Publishing. 
Schou, P., 2000. Polluting non-renewable resources and growth. Environmental and Resource Economics 16 (2), 211-227.

Seierstad, A., Sydsaeter, K., 1987. Optimal control theory with economic applications. North-Holland Amsterdam.

Tsur, Y., Zemel, A., 2005. Scarcity, growth and R\&D. Journal of Environmental Economics and Management 49 (3), 484-499.

\section{A. Appendix}

The complementary slackness conditions for research and capacity investments are

$$
\begin{aligned}
& \zeta_{a} \geq 0, \zeta_{a} a=0, \\
& \zeta_{q} \geq 0, \zeta_{q} q=0, \\
& \zeta_{\bar{I}} \geq 0, \zeta_{\bar{I}}(\bar{I}-a-q)=0 .
\end{aligned}
$$

The maximization of the Hamiltonian $H=U(c)+\lambda[F(K, x)-C(R)-B(A) b-a-q-$ $c]-\tau R+\kappa a+\theta q$ with respect to $a$ and $q$ gives

$$
\begin{array}{llll}
a=0 & \& & q=0, & \text { if } \lambda>\kappa \text { and } \lambda>\theta, \\
0 \leq a \leq \bar{I} & \& & q=0, & \text { if } \lambda=\kappa \text { and } \lambda>\theta, \\
a=0 & \& & 0 \leq q \leq \bar{I}, & \text { if } \lambda>\kappa \text { and } \lambda=\theta, \\
0 \leq a+q \leq \bar{I}, & & & \text { if } \lambda=\kappa \text { and } \lambda=\theta, \\
a=\bar{I} & \& & q=0, & \text { if } \lambda<\kappa \text { and } \kappa>\theta, \\
a=0 & \& & q=\bar{I}, & \text { if } \kappa<\theta \text { and } \lambda<\theta, \\
a+q=\bar{I}, & & & \text { if } \lambda<\kappa \text { and } \kappa=\theta .
\end{array}
$$

Optimal investments are determined by the complementary slackness conditions (A.1) (A.3), (10), (11), and (A.4). Suppose (A.4)(iv) holds, so that the sum of research and capacity investments is singular. Due to (A.1) and (A.2), $\zeta_{a}=\zeta_{q}=0$. Furthermore, $\zeta_{\bar{I}}=0$, so that (10) and (11) hold. The values of $\zeta_{i}, i=a, q, \bar{I}$ for the other 6 cases can be deduced in a similar way. 